\title{
Ética militar en el marco de guerras de quinta generación: propuestas teóricas para reestructurar la educación en instituciones militares ${ }^{1}$
}

https://doi.org/10.21830/9789585377134.07

\author{
Oscar Felipe Barrera Herrera ${ }^{2}$ \\ Jairo Andrés Hernández Cubides ${ }^{3}$ \\ Yesid Eduardo Ramirez Pedraza \\ Escuela Superior de Guerra "General Rafael Reyes Prieto"
}

1 Este capítulo presenta los resultados colaborativos de dos proyectos de investigación: (1) "Ética militar en entornos complejos de seguridad y defensa: lecturas y aportes desde la experiencia de las Fuerzas Militares de Colombia”, del grupo de investigación Masa Crítica, de la Escuela Superior de Guerra "General Rafael Reyes Prieto", Colombia, categorizado en B por Minciencias y con código de registro COL0123247, y (2) "Mujeres de arma, seguridad y defensa nacional. Un análisis desde sus percepciones", del grupo de investigación en Ciencias Militares, de la Escuela Militar de Cadetes "General José María Córdova”, Colombia, categorizado en B por Minciencias y con código de registro COL0082556. Los puntos de vista pertenecen a los autores y no reflejan necesariamente los de las instituciones participantes.

2 Magíster en Seguridad y Defensa Nacionales de la Escuela Superior de Guerra "General Rafael Reyes Prieto". Profesional en Ciencia Política y Gobierno de la Universidad del Rosario. Coordinador de Investigación de la Maestría en Seguridad y Defensa Nacionales de la Escuela Superior de Guerra "General Rafael Reyes Prieto", Colombia, y docente universitario. OrCID: https://orcid.org/0000-0003-3326-1138 Contacto: oscar.barrera@esdegue.edu.co

3 Magíster en Derechos Humanos y Derecho Internacional de los Conflictos Armados de la Escuela Superior de Guerra "General Rafael Reyes Prieto". Administrador público y especialista en Derechos Humanos de la Escuela Superior de Administración Pública de Colombia. Investigador de la línea Memoria y Conflicto del Instituto para la Pedagogía, la Paz y el Conflicto Urbano (IpAzud) de la Universidad Distrital Francisco José de Caldas y coordinador de Investigación de la Maestría en DD. HH. y Dica de la Escuela Superior de Guerra "General Rafael Reyes Prieto", Colombia. Orcid: https://orcid. org/0000-0002-6990-8637 - Contacto: jairo.hernandez@esdegue.edu.co

4 Magíster en Seguridad y Defensa Hemisférica del Colegio Interamericano de Defensa de Washington, D. C. Especialista en Dр. Hн. y Diн de la Escuela de Derechos Humanos. Profesional en Ciencias Militares de la Escuela Militar de Cadetes "General José María Córdova" y en Gerencia de la Seguridad de la Escuela de Inteligencia y Contrainteligencia "BG. Ricardo Charry Solano". Director de la Maestría en Seguridad y Defensa Nacionales de la Escuela Superior de Guerra "General Rafael Reyes Prieto", Colombia, y docente universitario. OrCID: https://org/0000-0002-0509-0995 - Contacto: yesid. ramirez@esdegue.edu.co 


\section{Resumen}

Este capítulo de libro analiza el concepto de ética militar en el marco de las guerras de quinta generación. La irrupción de este tipo de guerras, caracterizadas por la multiplicidad de actores, medios y capacidades, ha trascendido en la identificación del término por parte de las Fuerzas Militares, entendiendo que el contexto en el que se desarrollan son escenarios completamente nuevos. La investigación se realizó con un enfoque cualitativo de carácter descriptivo, mediante el cual se hace una conceptualización sobre la ética militar con el fin de esclarecer su definición profundizando en el concepto de guerra y su evolución de acuerdo con los preceptos de William Lind (2004), de tal manera que se introduce al lector en el concepto y se relaciona la ética militar y su importancia en el campo de las guerras de quinta generación. Finalmente, se establece la relevancia de repensar la educación en las instituciones militares en aras de fortalecer los procesos de comprensión de las dinámicas cívico-militares derivadas de las guerras de quinta generación.

Palabras clave: defensa; educación; ética militar; guerras de quinta generación; seguridad.

\section{Introducción}

\section{La ética y su aplicación en el ámbito castrense}

La ética se ha consolidado como el estudio filosófico de la conducta humana. El corpus aristotélico $(1831)^{5}$ en su tres tratados morales: Ética a Nicómaco, Ética a Eudemo y Gran ética, así lo estableció. Desde una perspectiva etimológica, la ética procede del vocablo èthos, que significa carácter o modo de ser, que se deriva o se traduce por hábito, de manera que es posible establecer que el carácter o modo de ser al que se alude no es el temperamento o la constitución psicobiológica que se tiene por nacimiento, sino la forma de ser que se adquiere a lo largo de la vida y que está emparentada con el hábito, que es bueno en virtud o malo en vicio (Díaz, 2015).

Este concepto ha sido aplicado a todos y cada uno de los campos de la vida del ser humano, tanto en el ámbito personal como en el profesional, en aras de brindar al individuo un margen de acción en el cual pueda definir entre el bien y el mal. Sin embargo, más allá de los tratados morales del corpus aris-

5 Fecha a la que corresponde la edición prusiana de Immanuel Bekker. 
totélico, existen diversas obras, como la ética de Spinoza (2019), que centra el campo de la ética en función de la libertad de elegir como base fundamental del accionar del ser humano.

Ahora bien, como lo afirma la psicóloga Gloria Elena Betancur Jiménez (2016), magíster en Asuntos Humanísticos, la ética y la moral son conceptos que hoy en día están en el ojo del huracán, pues a su alrededor se han establecido creencias y afirmaciones que hablan en su nombre, sin saber con exactitud cuál es el marco de significancia que se tiene de la ética o a cuáles códigos morales hacen referencia.

Este fenómeno, de acuerdo con Betancur (2016), se observa en las dinámicas políticas y empresariales, en los debates académicos en los claustros universitarios, en periódicos y revistas, e incluso en espacios de menor escala como las reuniones de padres de familia. Hace un tiempo era un discurso de filósofos para filósofos, de la religión para los feligreses, pero hoy se ha convertido en un tema cotidiano y de preocupación en espacios públicos y privados (Añasco \& Vera, 2019).

En este sentido, los asuntos militares no han sido ajenos al concepto de la ética desde la época de Santo Tomás de Aquino, San Agustín y Francisco de Vitoria. Pocos siglos después del nacimiento de Cristo, Agustín de Hipona ya empezaba a hablar de una defensa legítima del Estado por parte de sus fuerzas en caso de una agresión de otro actor que llegara a perturbar la paz y la tranquilidad. Es importante resaltar un punto en relación con el desarrollo de la connotación del bien, representado en la paz y la tranquilidad, perturbado por el mal, materializado en la intención de un actor de generar una agresión a otro (López et al., 1999).

En la concepción de San Agustín, los ejércitos de los pueblos agredidos tenían una base moral que les permitía responder con fuerza ante los eventuales ataques de los que pudiesen ser víctimas, legitimando de una u otra manera el accionar violento o el uso legítimo de la fuerza por parte de sus tropas en aras de mantener la paz y la tranquilidad de su territorio (López et al., 1999). Sin embargo, fue tal vez Marco Tulio Cicerón, antes de Cristo, el primer autor en postular alguna idea de justicia en la guerra, al sostener que las guerras legítimas deben ser abiertamente declaradas, abrigar una causa justa y 
ser conducidas de manera justa. Sin embargo, ¿qué es justo en el marco de la guerra?

Para responder a este interrogante se puede recurrir a los planteamientos sobre la guerra justa hechos por San Agustín de Hipona, quien intentó conciliar las enseñanzas de Cristo con la defensa del Imperio Romano, que en ese marco contextual se encontraba en su ocaso y necesitaba ser defendido de las invasiones de los pueblos germanos que tendían al auge (Herrera, 2012). San Agustín (1994) justificó la guerra como el medio para conseguir la paz en los siguientes términos:

Quien considere en cierto modo las cosas humanas y la naturaleza común, advertirá que así como no hay quien no guste de alegrarse, tampoco hay quien no guste de tener paz. Pues hasta los mismos que desean la guerra apetecen vencer y, guerreando, llegar a una gloriosa paz. ¿Qué otra cosa es la victoria sino la sujeción de los contrarios? Lo cual conseguido, sobreviene la paz. Así que con intención de la paz se sustenta también la guerra, aun por los que ejercitan el arte de la guerra siendo generales, mandando y peleando. Por donde consta que la paz es el deseado fin de la guerra, porque todos los hombres, aun con la guerra buscan la paz, pero ninguno con la paz, busca la guerra. (San Agustín, 1994, p. 479)

Casi un milenio después, Santo Tomás de Aquino cuestionó los planteamientos hechos por San Agustín de Hipona en la ardua labor de poner en orden el conocimiento de la época y brindar una base teológica, que materializó en su obra Suma teológica. De acuerdo con Ángela María Herrera (2009), en esta obra, considerada como una de las más importantes de la filosofía cristiana, Santo Tomás (1950) afirma que se requieren tres cosas para una guerra justa.

En primera medida, Santo Tomás sostiene que la potestad de declarar la guerra no pertenece a una persona privada, porque puede exponer su derecho ante juicio del superior, teniendo en cuenta que a estos les corresponde defender el interés público de la ciudad, del reino o de la provincia a ellos sometidos. En segundo lugar, es necesaria la existencia de una justa causa, es decir, que quienes sean impugnados en el marco de la guerra tengan per se merecimiento alguno a esa impugnación. Finalmente, se requiere que sea recta la intención de quienes hacen la guerra, es decir, que se intente o se promueva el bien o que se evite el mal (Aquino, 1950). 
Posteriormente, Vitoria y Pereña (1989), en relación con los derechos de la Corona española en los territorios conquistados de América y los derechos de los habitantes de aquel continente, amplían la noción de la guerra justa y establecen con mayor claridad la distinción entre el ius ad bellum (derecho sobre el empleo de la fuerza) y el ius in bello (derecho de la guerra), teniendo en cuenta los preceptos de San Agustín y de Santo Tomás. En este sentido, Vitoria y Pereña (1989) sostienen que lo que era lícito en las leyes naturales y escritas no deja de serlo en la ley evangélica, y agregan:

Y porque no puede ponerse en duda la licitud de la guerra defensiva, puesto que es lícito repeler la fuerza con la fuerza, se prueba también la licitud de la guerra ofensiva, esto es, de aquella en la cual, no solo se defienden o se reclaman las cosas, sino que además, se pide satisfacción por una injuria recibida. (Vitoria \& Pereña 1994, p. 12)

De acuerdo con lo anterior, Francisco de Vitoria legitima la guerra ofensiva y no simplemente la defensiva, porque de acuerdo con San Agustín no habría paz ni seguridad en la república si no se mantiene a raya el enemigo con el temor de la guerra. Es decir, en términos modernos, empleando la disuasión como mecanismo para repeler al enemigo.

De este modo, para sentar un precedente respecto al concepto de guerra justa del cual se había empezado a hablar 1.500 años atrás (de su época) con Marco Tulio Cicerón, el teólogo español establece como única causa justa la injuria recibida y niega como causas justas las controversias por diversidad de religión, el deseo de ensanchar el propio territorio y la gloria o el provecho particular del príncipe, siendo estas las razones por las cuales se libraron la mayoría de las guerras en su época y hasta hace poco tiempo atrás (Herrera, 2012). Sobre la causa justa hace una anotación más: "No basta con cualquier injuria para declarar una guerra: debe ser una injuria que merezca una guerra, en la cual habrá devastación, incendios y matanzas" (Vitoria \& Pereña, 1989, p. 131). En este sentido, de acuerdo con las reflexiones de San Agustín, pasando por Santo Tomás de Aquino hasta Francisco de Vitoria, se puede observar una transformación del concepto de lo que es la guerra justa. Dicha transformación, hasta este punto, podría resumirse como una noción desde la cual la guerra es justa dependiendo de la perspectiva en la cual se analice. Algo similar 
pasa con el concepto de ética vista como el hábito de lo bueno en virtud o de lo malo en vicio. Sin embargo, gracias a la creación de la Declaración Universal de los Derechos Humanos, la evolución del Derecho Internacional Humanitario (Dıн) y los ordenamientos jurídicos internos, se han establecido las bases para la consolidación de lo que hoy podría denominarse como ética militar.

De acuerdo con Juan Moliner González (2018), general de división del Ejército del Aire y subdirector del Instituto Universitario General Gutiérrez Mellado, se entiende que existe una ética universal, que está fundada en los valores y principios esenciales recogidos en la Declaración Universal de los Derechos Humanos, convertida en código moral de aspiración global. Atendiendo a la enunciación de Moliner (2018), la consolidación del concepto de ética militar podría concebirse para evitar no tanto un choque entre civilizaciones, teniendo en cuenta la diversidad de perspectivas y creencias de las sociedades globales, sino como un marco de valores que tienen la necesidad de realizar esfuerzos de entendimiento entre las civilizaciones que lleven a

conciliar la riqueza que supone la variedad de culturas con aquello que las reúne a todas y las unifica, a saber, una cultura mediática de alcance planetario como herramienta y medio de difusión de conocimientos. Solamente así se podrá dar respuesta a las aspiraciones comunes que comparten todos los pueblos del planeta. (Moliner, 2018, p. 9)

Respecto a lo anterior, se puede afirmar que el concepto de ética visto desde la filosofía del corpus aristotélico, sus tratados morales y la concepción de ética militar, se ha venido consolidando como una base sólida o un conjunto de principios que tienen por objetivo armonizar el comportamiento de los miembros de los ejércitos con las sociedades globales. Lo anterior, con el respaldo de un andamiaje jurídico que enmarque esos comportamientos universales en unos parámetros claros que delimitan el accionar de las Fuerzas del orden a nivel mundial.

En este punto es importante tener en cuenta que las guerras de la época de Cicerón, San Agustín, Santo Tomás y Francisco de Vitoria no son las mismas. Gracias a la evolución de la tecnología se han forjado nuevos escenarios que ni siquiera fueron imaginados hasta hace por lo menos cincuenta años. Para explicar este escenario, es necesario llevar a cabo un análisis de la evolución de 
las guerras con el propósito de comprender a qué se enfrentan las sociedades modernas en la actualidad. Para ello, se hará un análisis de las características de la evolución de las guerras desde el nacimiento de los Estados modernos con la paz de Westfalia (Elliott, 1999), teniendo en cuenta las categorías analíticas de William Lind (1985) y su estudio de la transformación de las guerras en su obra Maneuver Warfare Handbook, hasta el campo de las guerras de quinta generación.

\section{La evolución de las guerras y los nuevos campos de batalla}

La guerra se ha caracterizado, de acuerdo con autores como Carl von Clausewitz (1989), como la continuación de la política por otros medios. En este entendido y tomando el concepto de política como las meras relaciones de poder en el marco de las dinámicas estatales, se puede inferir que la guerra ha sido el medio con el cual los Estados se han forjado y han buscado la proyección de sus intereses en el sistema internacional.

Es menester enunciar que atendiendo a las dinámicas que ha definido el autor respecto a la guerra, esta ha sufrido una serie de cambios dentro de la trazabilidad diacrónica en relación con su capacidad de desarrollo; es decir, la guerra ha ido evolucionando en la medida en que las capacidades tecnológicas de la humanidad se han transformado. Teniendo en cuenta lo anterior, William Lind (2004) se dio a la tarea de analizar la evolución de las guerras delimitando su estudio desde el ańo 1648, época en la cual surge el Estado moderno, pasando por cuatro generaciones que desembocan en la guerra de quinta generación, la cual ha sido definida por Rey Alderman (2015) como la guerra sin contacto.

\section{La primera generación de la guerra}

Lind (2004) describe que la primera generación contempla desde 1648 hasta 1860, época en la cual se desarrolló y consolidó el concepto del Estado. Esta generación, derivada de los avances armamentísticos del Renacimiento, apropia nuevas formas de relaciones sociales que repercuten directamente en los nuevos modelos de ejército: la guerra de líneas y columnas tácticas, las cuales estaban diseñadas con poder de fuego en la primera línea para avanzar 
rápidamente en los territorios en los que se libran las batallas. Su potencia de fuego era menor debido a que únicamente las primeras filas podían disparar a las tropas enemigas.

Además del uso de armas de fuego y la formación de ejércitos profesionales que responden a una lógica estatal, en el marco de estas guerras se creó una cultura militar del orden, en la cual aparecen diversos elementos que distinguen a un militar de un civil, como los uniformes y las graduaciones. Las características de esta primera generación de la guerra, al día de hoy, se han consolidado como una de las bases más importantes en el marco de lo que se ha denominado la ética militar. La distinción entre un cuerpo legal armado con mando responsable encargado de velar por la seguridad del Estado respecto a la población civil hizo necesario que se creara ciertos parámetros derivados de la paz de Westfalia que supusieron modificaciones en las bases del derecho internacional, las cuales estaban encaminadas a lograr un equilibrio europeo que impidiera a unos Estados imponerse a otros (Lind, 2004).

Con el Renacimiento, cambiaron las dimensiones físicas de la guerra, el tamaño de los ejércitos y la escala de las operaciones aumentó, así como lo hizo la potencia de fuego y se desarrollaron nuevas técnicas de fortificación. El crecimiento de los ejércitos y de la duración de las campañas provocó un incremento de la fiscalidad y la elaboración de políticas de guerra.

\section{La segunda generación de la guerra}

Está basada en los logros y las capacidades derivados de la revolución industrial y la potencia de fuego lograda con las innovaciones tecnológicas de la época. Esta segunda generación está ejemplificada en la Primera Guerra Mundial (1914-1918), la cual se basó en la capacidad industrial y de transportes. Mientras que en las guerras de primera generación se combatía por intereses y sobre ellos cabía un compromiso, ahora eso ya no era posible porque el combate se sustenta también sobre las ideas y se ha movilizado al pueblo en torno a ellas (Lind, 2004).

Otro aspecto importante de esta segunda etapa es la creación de trincheras, las cuales fueron diseñadas con el fin de proteger a los soldados de la potencia del fuego enemigo. Como se mencionó anteriormente, el legado de 
la primera generación tipificada por Lind (2004) se mantuvo, toda vez que el orden, los procesos y los procedimientos en el marco de la confrontación se mantuvieron atendiendo a la obediencia, producto de la profesionalización y los ejércitos, como baluarte fundamental del éxito de los ejércitos en los escenarios bélicos. El objetivo principal de los combates sigue siendo el ejército enemigo.

En el marco del fin de la Primera Guerra Mundial, de la mano del presidente de los Estados Unidos Woodrow Wilson nació un modelo del cual se deriva lo que hoy día se conoce como el idealismo de las relaciones internacionales, mediante el cual se presentó una serie de puntos que tuvieron por objeto dar por terminada la Primera Guerra Mundial y, sobre todo, evitar que otra guerra de la misma magnitud se llevara a cabo en el futuro (Bruera, 2018). Este idealismo enmarcado en las relaciones internacionales configuró una nueva ética en el contexto del sistema internacional, la cual fue esbozada por Woodrow de la siguiente manera:

Lo que pedimos es que el mundo sea un lugar apto y seguro para vivir, y, en particular, para todo país amante de la paz que, como el nuestro, desee vivir su propia vida, decidir sus instituciones, recibir garantías de justicia y tratos justos por parte de otros pueblos, así como contra la fuerza y la agresión egoísta [...]. Nuestro programa es la paz mundial y este programa es el único posible. (Wilson, 1918, p. 2-3)

\section{La tercera generación de la guerra}

Tras la Segunda Guerra Mundial, paulatinamente se fue produciendo un cambio en los modos de producción y algunas sociedades disminuyeron el peso del sector industrial en beneficio del sector servicios; la consecuencia fue la progresiva aparición de una economía basada en los conocimientos más que en las materias primas, el esfuerzo físico o el desarrollo industrial. Este cambio en las sociedades trajo una modificación en la forma de hacer la guerra, que ahora es realizada por tropas altamente especializadas y en la que la tecnología ocupa un lugar destacado.

La tecnología vuelve a desempeñar un papel preponderante, pues el principio de esta generación radica en la rapidez, por lo cual se establece 
el concepto de Blitzkrieg ('guerra relámpago'), que se basa en la guerra de maniobra a través de blindados y aviones, ejemplificada en el ejército alemán de la Segunda Guerra Mundial. En esta generación la tecnología no está direccionada a mejorar la potencia de fuego y el desgaste, sino en la velocidad, la sorpresa y la dislocación mental orientadas a rodear y colapsar al enemigo evitando el contacto directo. Por ejemplo, se llevan a cabo ataques a puntos estratégicos del enemigo — como las comunicaciones - enfocados en aislar sus fuerzas y bloquear una respuesta sostenida conjunta. En este sentido, la superioridad tecnológica es crucial para el éxito.

\section{La cuarta generación de la guerra}

La característica principal es la pérdida del monopolio del uso de la fuerza por parte de los Estados, lo cual claramente deriva en la pérdida del monopolio sobre la guerra, toda vez que emergen una multiplicidad de actores no estatales, como los grupos terroristas y las guerrillas. En este nuevo contexto estratégico, la victoria no está en un campo de batalla porque tales acontecimientos ya no tienen lugar como en las tres primeras generaciones.

Ahora la victoria se consigue, por ejemplo, al influir y convencer a la sociedad (opinión pública) en la búsqueda u objetivo de ganarse el apoyo de las personas, de manera que el centro de gravedad del conflicto deja de ser las bajas de las tropas enemigas como indicador del éxito o el fracaso en la guerra. Para lograr esta influencia hay dos elementos fundamentales que deben confluir para penetrar en la mente de las personas: la propaganda y el uso de acciones terroristas orientadas a infundir miedo en una sociedad objetivo y así condicionarla a las exigencias mediante el terror.

Según Lind (2005), en su artículo "Comprendiendo las guerras de cuarta generación”, esta fase está caracterizada por un retorno al mundo de culturas y no simplemente Estados en conflicto. Para el autor, en el marco de esta confrontación Occidente enfrenta al más antiguo y firme adversario del mundo cristiano occidental: el Islam, pues gracias a la interconectividad

6 El Blitzkrieg (literalmente, 'guerra relámpago') es un nombre popular para una táctica militar de ataque que implica un bombardeo inicial, seguido del uso de fuerzas móviles atacando con velocidad y sorpresa para impedir que un enemigo pueda llevar a cabo una defensa coherente (Blitzkrieg vs. Batalla profunda) (Ureña, 2012). 
global, a través de la migración masiva ha logrado un asentamiento de importantes magnitudes en grandes ciudades del mundo occidental, que puede ser tan peligroso como la invasión que emplea un ejército de un Estado en el marco de una guerra convencional.

\section{La quinta generación de la guerra}

El concepto de guerra de quinta generación tuvo su origen alrededor del año 2005. Roy Alderman (2015) la califica como una guerra sin contacto y silenciosa, que está fundamentada básicamente en el aprovechamiento masivo de los medios cibernéticos y en el dominio de la mente (González, 2017). Es justo en este punto cuando se empieza a ver un nuevo escenario que no había sido explotado de la primera a la tercera generación de la guerra y que en la cuarta empezó a desempeñar cierto papel. Sin embargo, en el marco de las guerras de quinta generación, el campo de los medios cibernéticos recobró gran importancia, de manera que, por razones obvias, las guerras se han transformado y han llegado a librarse en campos nunca antes vistos por los soldados del mundo.

En este contexto, los ejércitos de los Estados se vieron abocados a enfrentar nuevos retos en un campo de batalla nunca antes explorado y de todas formas desconocido, campo para el cual los marcos jurídicos internacionales y los ordenamientos jurídicos no estaban preparados para enfrentar, razón por la cual fue necesario empezar a fortalecer los organismos de comunicaciones y de inteligencia de las fuerzas de seguridad global en las nuevas dinámicas de esta guerra silenciosa (González, 2017).

El corte de esta generación radica en el uso de la información en beneficio propio. Esta información es extraída a través de vías tecnológicas y tiene por objetivo generar una ventaja comparativa respecto al enemigo recabando un poco en lo que surgió de las guerras de cuarta generación como una estrategia de influencia en la opinión pública. Cuando una de las partes logra hacerse al monopolio, este poder le otorga por sí mismo una posición de ventaja absoluta sobre el oponente, toda vez que los beneficios derivados de esa capacidad de influencia sobre la opinión pública la hará portadora de la voluntad de las masas, de tal manera que tiene como resultado la capacidad de poner la balanza a su favor (Otero, 2012). 
En resumen, de acuerdo con lo anterior, cuando ocurren ese tipo de escenarios, tener la balanza a favor otorga un poder supremamente valioso en el marco de cualquier confrontación, el cual consiste, por una parte, en la capacidad de influir en la toma de decisiones alrededor de los actores inmersos en la guerra, y, por otra, generar confusión e incertidumbre en las tropas enemigas, de tal manera que puede afectar, por supuesto, sus capacidades de mando y control.

El paso de la guerra de cuarta a la quinta generación rompe un esquema que basaba la intensidad de las guerras en el poder de fuego y en las capacidades armamentísticas de las partes involucradas. En el marco de este nuevo escenario silencioso se observan unas dinámicas que inevitablemente implican un reto de grandes dimensiones para todos los ejércitos del mundo. Este hecho hace muy necesario definir qué está bien y qué no está bien en el marco de estas nuevas guerras, lo cual nos llevará inevitablemente de vuelta al centro de gravedad de esta investigación: la ética militar.

\section{La ética militar en el campo de las guerras de quinta generación}

Entendiendo la ética militar como esa base de principios para el entendimiento de las Fuerzas del orden entre sí mismas y con los pueblos del mundo en medio de un conflicto, es necesario empezar a pensar en la ética militar como una necesidad imperiosa en el campo de las nuevas guerras.

La volatilidad y la falta de fronteras ante la ausencia de los espacios físicos en el marco de estas guerras han abierto un espectro sumamente amplio que ha generado cierta ambigüedad en el accionar de las fuerzas de los Estados. Lo relativamente nuevo de este tipo de conflictos y, sobre todo, lo sui generis, es que los espacios cibernéticos en los que se desarrollan han tenido como consecuencia la difícil comprensión de lo que está bien y lo que está mal (hablando en términos de ética).

De acuerdo con Luis Ignacio Gutiérrez Sierra (2009), profesor de la Pontificia Universidad Javeriana de Bogotá, Colombia, y doctor en Ciencias de la Comunicación de la Universidade de Vale do Río dos Sinos, Brasil, la revolución cibernética, 
entendida como la mutación tecnológica que va desde la acumulación y el flujo constante de información en la red hasta la realidad virtual con sus múltiples posibilidades de autorrepresentación y simulación digitalizada, "ha transformado la vida de las personas y ha modificado sustancialmente las maneras y los modos como nos relacionamos, nos informamos, trabajamos, jugamos, aprendemos, negociamos, representamos e interactuamos. (Gutiérrez, 2009, p. 94)

Asimismo, según Gutiérrez (2009), en la sociedad han ido creciendo paralelamente una serie de cuestionamientos éticos respecto a los alcances, los riesgos y las posibilidades que se derivan del uso de la tecnología sobre el manejo de la información, la cual es un elemento vital en el marco de las guerras de quinta generación, dado que la obtención de esta significa un paso hacia la victoria, pues como reza un dicho común, y sobre todo en las nuevas dinámicas del mundo de la tecnología, la información es poder.

Continuando con la idea del doctor Gutiérrez (2009), es importante resaltar que en la actualidad en la comunidad del ciberespacio aún ronda la idea de que "quien actúa en el ciberespacio se mueve dentro de una zona inmune a la ética”, donde se es completamente libre y autónomo en sus comportamientos (Gutiérrez, 2009, p. 95). Esa idea es justamente el gran peligro al que se enfrenta el campo de la ética en el marco de las guerras de quinta generación, pues ante la multiplicidad de escenarios en los cuales un espacio no físico es predominante, se genera un vacío que sin duda cambia la forma en la que los humanos ven el mundo y las consecuencias de los actos que allí se cometen.

En este sentido, es supremamente importante establecer un nuevo concepto de ética militar orientado a llenar los vacíos y superar la incertidumbre que se desarrolla en el ciberespacio, con el fin de descontinuar la idea de que quien actúa en el ciberespacio lo hace en un espacio donde no hay lugar a los comportamientos éticos.

La situación derivada de la pandemia producto de la Covid-19 en el año 2020 ha dado aún más relevancia a este tipo de espacios, pues saliendo un poco de las dinámicas de conflicto propias de las guerras que se han desplazado al ciberespacio, se ha convertido en un escenario en el cual la sociedad del siglo XXI ha empezado a interactuar a nivel personal, laboral y familiar. En este sentido, pasar de la cibernética a la ciber-ética, como lo postula Gutiérrez 
(2009), es una necesidad imperante a nivel general, pero se hace menester en el área castrense toda vez que de allí se derivan los principales retos en materia de seguridad y defensa de la actualidad.

En este sentido, resulta fundamental repensar el concepto de ética militar y aplicarlo a las nuevas dinámicas globales que han cambiado la forma como se relacionan y, por ende, se enfrentan unos con otros, lo cual está enmarcado en el concepto de las guerras de quinta generación. Desde la academia, es posible plantear un curso de acción que lleve a establecer las bases de esta nueva perspectiva de la ética militar, con el fin de aportar a las Fuerzas elementos de base que conlleven la delimitación del comportamiento de sus integrantes orientados por esta lógica vigente. Si bien con la pérdida del monopolio de la guerra por parte de los Estados en el marco de la cuarta generación propuesta por Lind (2004) los enemigos del orden han actuado sin ningún precepto ético, los Estados y sus respectivas Fuerzas están obligados a respetar determinadas directrices y puntos con el fin de actuar bajo el marco de la legalidad, concepto relacionado directamente con la ética y la moral.

Sin embargo, en este punto es importante traer a colación la perspectiva del Mayor General en uso de buen retiro de la Fuerza Aérea de los Estados Unidos, quien establece que por encima de las dudas sobre la aplicación de regímenes legales y costumbres éticas para un ciberescenario particular, está la cuestión más amplia de si se debería aplicar restricciones, lo cual abre un debate interesante puesto que se tiene la creencia de que los esfuerzos para aplicar la ley dificultan la defensa de la seguridad del Estado. Al respecto, Bermúdez (2014) reconoce:

El respeto del Estado de derecho es especialmente importante en el ámbito ciberespacial porque casi todos los expertos concuerdan en que la confrontación de la amenaza requiere de la cooperación de otros países a fin de rastrear y neutralizar las ciberamenazas en tiempo de paz o de guerra. Las naciones vitales para este esfuerzo, incluyendo especialmente las grandes democracias del mundo, indudablemente no estarían dispuestas a cooperar con ningún país que rechace límites en las operaciones militares, ciberespaciales o de cualquier otra forma. (p. 14) 
Esta postura es abiertamente respaldada por reconocidos académicos como Chris T. Antoniou y Michael Riesman (1994), quienes señalan que por más loable que sea el objetivo de una guerra, o una ciberguerra, si esta se lleva de manera injusta o inhumana va a perder el apoyo de la sociedad.

\section{Propuesta para el fortalecimiento de la ética militar en el campo de las guerras de quinta generación}

En el presente apartado es preciso proponer medidas de fortalecimiento de la ética militar en el campo de las guerras de quinta generación, ya que en el marco conceptual se ha problematizado la transposición de la ética a un lugar difuso y se ha reconocido el importante valor de la ética en el marco social y militar. De esta manera, se pretende hacer un acercamiento pedagógico que permita crear una propuesta de carácter teórico, en la cual se trabajen posibles autores para incorporarlos en el marco de la formación educativa de la población militar y problematizar de esta forma el contexto de las guerras de quinta generación. Así pues, el apartado tiene dos secciones fundamentales: la primera gira en torno a la problematización de la ética en el marco de la tecnología, en tanto que la segunda se refiere a los mecanismos pedagógicos para hacer una sensibilización sobre el papel de la ética en el contexto militar.

\section{Ética militar en el marco del control tecnológico}

Los cambios sociales que ha generado la globalización afectan seriamente la civilización y específicamente a la nación. Asimismo, los desequilibrios existentes en el orden político, económico y cultural también han configurado las manifestaciones de la guerra, como se pudo ver en el campo de la quinta generación. El nuevo paradigma cambia su estructura fundamental del duelo a una serie de dinámicas estratégicas que inciden en la reconfiguración del seguimiento y las tácticas hostiles de vigilancia.

En el año 2001, luego del fenómeno histórico del 11 de septiembre, George Bush afirmó que "los Estados Unidos se lanzarán a un nuevo género de guerra. Una que requiere por nuestra parte una cacería internacional del hombre" (The White House, 2003). Este fue el punto de partida para que los 
Estados Unidos creara en una década una nueva categoría de violencia estatal, sustentada en la cacería humana militarizada. Haciendo referencia a George A. Crawford, un abogado y político estadounidense, Poole (2009) planteó en un informe para la Joint Special Operations University que la cacería humana como fundamento de la política militar es uno de los pilares de la estrategia indispensable en la construcción del futuro.

De esta manera, contra la definición clásica de la guerra, se abandona la idea de la confrontación lineal fundamentada en el duelo y se apropia un paradigma asociado con el ejercicio de la caza, donde los personajes cazador y fugitivo se entienden como macrosujetos ${ }^{7}$ que encarnan unas prácticas culturales, económicas, sociales, políticas y demás, y donde el poder es ejercido a mayor o a menor escala. En este escenario, el cazador tienen como objetivo capturar a su fin por medio de la identificación y la localización del enemigo. Al respecto, es menester mencionar que el nuevo rol del cazador, en el marco de la quinta generación, supone todo un trabajo de detección por medio de la tecnología y de herramientas como vigilancia aérea, huellas cartográficas e interceptación. Por su parte, el fugitivo centra su atención en no ser alcanzado. Esta analogía problematiza un aspecto de la ética militar que se refiere al poder invasivo de las actividades diarias, el cual se sustenta en el derecho a la persecución, intentando ya no ocupar un territorio, sino dominarlo por medios de control.

Problematizar las dinámicas de la cacería humana, de acuerdo con Ávila (2006), permite reconocer la guerra desde una perspectiva foucaultiana y establecer cómo por medio del ejercicio del control, la vigilancia y la localización se previenen ataques de posibles enemigos, donde el poder se ejerce y marca un punto de partida situado en el marco cultural y político en función de los medios militares. En palabras de Ávila (2006),

el mecanismo del poder es la represión (hipótesis de Reich), a la vez observamos que el trasfondo de la relación de poder es el enfrentamiento armado de las fuerzas que se disputan el control del poder (hipótesis de Nietzsche). Estas dos hipótesis no son mutuamente excluyentes, sino que se conjugan y

7 Macrosujetos se puede entender mediante el ejemplo de que a una ciudadanía se le puede establecer características de sujetos que repliega, reproduce, produce subjetividades e intersubjetividad. Además, porque es regulado y ejerce el poder, en mayor o menor escala, de acuerdo con Foucault (2001). 
se complementan, ya que la represión puede definirse como corolario político de la guerra. En la relación guerra-poder, habría que necesariamente referirnos a la guerra para ver cómo funciona el poder; ya que subyace la idea de que el poder tiene como misión fundamental la defensa de la sociedad, es decir, habría que dar por aceptado que esta está organizada de una manera tal, que unos pueden defenderse de los otros o defender su capacidad de dominación contra la posibilidad de rebelión de otros. (Ávila, 2006, p. 224)

Las nuevas medidas de control dentro del marco militar problematizan el concepto de la ética militar, dado que, al reconocer las amenazas, se implementa un análisis de las formas de producción cultural de la población, por medio de nodos tecnológicos como el que ofrecen las redes sociales, las cámaras de vigilancia, los drones y otros elementos. La información capturada constituye las prácticas de la vida en general, de manera que si a una persona que opere por fuera del margen de la normalidad se le considera un enemigo, se traza el seguimiento con el fin de reconocer sus lugares habituales, gustos y núcleos familiares, entre otros, para así reducir la amenaza de una conducta irregular.

$\mathrm{Al}$ reconocer las nuevas formas de desarrollo en la guerra, resulta importante entonces establecer sus cuatro elementos básicos, según Liang y Xiangsui (2002; citados por Álvarez et al, 2017): (1) los nuevos dominios del conflicto; (2) la naturaleza cambiante de los adversarios; (3) la naturaleza cambiante de los objetivos, y (4) la naturaleza cambiante de la fuerza. Este último elemento aborda la naturaleza de la guerra, es decir, su esencia misma como ejercicio de la voluntad humana en la aplicación de la fuerza, mientras que los otros se refieren a los medios de la guerra. Liang y Xiangsui (2002; citados por Álvarez et al., 2017) sostienen que hasta el presente no se han cuestionado los elementos básicos de los dominios, los adversarios, los objetivos y la fuerza:

No obstante, el problema de la guerra en la era posmoderna deriva de los procesos-productos de los impactos políticos, económicos, sociales y tecnológicos de la era de la información y de la globalización, y porque la conducción de la guerra se ha extendido más allá de las fuerzas estrictamente militares, estos elementos han cambiado profundamente, lo que exigiría una nueva comprensión de ellos. (Liang \& Xiangsui, 2002; citado por Álvarez et al., 2017) 
El papel de la ética militar trasciende entonces a un marco vital dentro de la formación ciudadana, entendiendo que el fenómeno de la globalización es irreversible y que únicamente una formación moral y ética descentralizada contribuye a la deconstrucción de las fuerzas de dominio en relación con la etapa de la información. En este sentido, es menester reconocer los puntos de fuga que permiten situarse en el nuevo campo de batalla: el internet. A continuación, se genera una propuesta teórica que permite formas de reconocimiento decolonial y descentralizado en el marco de la tecnología con el fin de atribuir a la población ciudadana un lugar protagónico en la guerra, la cual ha trascendido a unas escalas inimaginables y de la cual han sido víctimas de los elementos mencionados.

\section{La quinta generación en el marco de la educación}

Como se ha mencionado, este capítulo centra sus esfuerzos en generar una propuesta teórica sustentable en el marco de la ética militar respecto al tema de las guerras de quinta generación. Para lograr dicho objetivo, en primer lugar, se propone que la formación tenga lugar en el marco institucional de la militancia y desde el punto de vista descentralizado, decolonial y propositivo, además de que diseñe su trazabilidad en temas de reconocimiento social. En un segundo lugar, se afianza esta propuesta en la aplicación de las tecnologías de la información y las comunicaciones (Tic) en el modelo educativo, con el fin de que sea consecuente con el contexto de la quinta generación y atienda las problemáticas que presenta este concepto en la globalización.

Una de las aplicaciones de la enseñanza ética es conceder espacios de meditación en torno al comportamiento humano, personal y en grupo en una sociedad, a fin de reconocer y cimentar la responsabilidad que tiene una persona frente a sí misma y los otros, con el fin noble de que se lleve a la práctica la transparencia de los actos, se asuma la responsabilidad en todo y se posibilite la toma de elecciones para el cultivo de la raza humana. De esta forma, se espera que la persona actúe continuamente dentro de la justicia y el respeto, acate las reglas que le hagan bien como sujeto y como miembro de una sociedad incluyente y multicultural, además de que acate los principios que rigen el Derecho Universal Humanitario. 
La ética, como se dijo en su momento, tiene un carácter polisémico y cambiante. Por lo tanto, en el marco de su enseñanza, la problematización de este concepto se traslada al análisis de la realidad humana, lo cual permite cuestionar la formación de escuelas, universidades e instituciones del Estado que, comprometidas con el pueblo, forman parte del mito fundacional de la civilización, como son la policía y el ejército. Ahora bien, las instituciones fundamentales (iglesia, ejército, corpus político, entre otros) que se desarrollan de la mano del crecimiento de las ciudades también han sido blanco de desacatos que ponen en punto de mira la ética profesional por la corrupción, el abandono y la invisibilidad de las comunidades vulneradas, entre otros ejemplos.

Este contexto lleva a que sea necesario replantear la ética militar desde la educación, entendiendo que la misión de las instituciones armadas del Estado fundamentan su misión en proteger y garantizar la soberanía de la nación, su seguridad y la democracia. Resulta fundamental pensar el marco propositivo atendiendo a faltas graves en las que han incurrido las Fuerzas Armadas y que son de conocimiento público: los falsos positivos, nexos con narcotraficantes, asesinatos y demás hechos atroces que vulneran los Derechos Humanos (Dр. Нн.) y el Derecho Internacional Humanitario (Dıн). Es decir, faltas graves a los Convenios de Ginebra del 12 de agosto de 1949.

Con el convencimiento de que no existe otro camino más que el de la educación para erradicar los abusos de poder en el contexto militar, el primer problema que plantea la propuesta de marco teórico es transformar el currículo. De acuerdo con Lozano y Parra (2015), "es imprescindible un cambio en el currículo que permita asumir conscientemente la importancia de la ética en la formación de las Fuerzas Militares y utilizar enfoques modernos de la enseñanza, a partir de una sólida base metodológica y didáctica" (Lozano \& Parra, 2015, p. 21).

En este sentido, el primer foco propositivo tiene que ver con el problema de la representación de las instituciones hegemónicas, en este caso militares, en el imaginario colectivo. Por eso, la propuesta teórica se debe pensar desde el lugar de enunciación que tienen las Fuerzas Militares, cuya población constituye un grupo hegemónico dominante, por cuanto forma parte del Estado, y ejerce un poder sobre otras poblaciones. Según explica la escritora belga 
Chantal Mouffe (1991) en su texto "Hegemonía e ideología en Gramsci", el concepto de sector hegemónico permite desligarse de la mirada determinista en la que se encontraba el poder en lecturas anteriores, como las de Althusser (1988), así como reformular la idea de Foucault de que el poder es ejercido y, por tanto, está en todas partes.

En contraste, la interpretación que hace Mouffe (1991) del concepto es que el poder se sitúa en los sectores hegemónicos y que esta ubicación o reconocimiento será de vital importancia para entender cómo se configura y cuáles son los procesos que debe seguir un sector para ejercerlo. Al respecto, cabe señalar que este apartado sigue la interpretación de Mouffe (1991) sobre las lecturas de Gramsci, de manera que el concepto de hegemonía se considera el núcleo problemático en el proceso de adquisición del poder.

Gramsci fundamenta su teoría en el concepto de hegemonía, a la cual describe como un consenso constituido por una compleja relación de fuerzas generada por intelectuales orgánicos, quienes responden a objetivos económicos, políticos, intelectuales y morales, mediados por la ideología, que tiene una forma material. En este punto es importante destacar la influencia que tuvo Althusser (1988) en la construcción de dicha tesis y el diálogo que establece Mouffe (1991) entre estos dos autores. La posibilidad de irrupción radica en que este concepto es entendido por el autor como el campo donde se funde la voluntad colectiva y la conciencia que permite luchar en el marco de prácticas sociales representadas por visiones del mundo, lo cual hace posible la unidad ideológica orgánica.

La ideología es fundamental en la obra de Gramsci, pues es en ella donde se desarrollan los procesos de intersubjetividad que dan al sujeto político un lugar de enunciación. La ideología es descrita por Gramsci como "el terreno donde los hombres se mueven, adquieren conciencia de su posición y luchan" (Mouffe, 1991, p. 198). En este sentido, el sujeto se sitúa de forma arbitraria en un espacio de prácticas sociales donde es producido por una ideología que no corresponde a un espacio de reproducción y de determinación, pero que sí está materializado por estructuras, aparatos e incorporación de prácticas que responden a un proceso de interpelación. Por lo tanto, la ideología brinda la posibilidad de generar una autonomía eficaz en relación con una voluntad colectiva que adopte los intereses de las clases que la hegemonía domina. 
Dentro de la categoría del bloque hegemónico que plantea Gramsci se puede situar al sujeto, quien materializa las ideologías y las difunde, de tal manera que es un agente social permeado por estas que a la vez puede configurar las dinámicas de los sectores hegemónicos por medio de un marco de acción. Esta mirada posmarxista del sujeto rompe con el principio reduccionista que sobrepone la problemática de la clase social sobre toda teoría y permite que se dé una reforma en la articulación del sistema hegemónico. Así pues, en el ejercicio de interpelación el sujeto recrea formas materiales que se irrumpieron por medio de la acción política (Mouffe, 1991, p. 195) que funde la unidad ideológica.

Es de vital importancia establecer algo que hasta el momento no se ha dicho y es que la hegemonía, al ser un proceso-resultado del consenso social, atraviesa el campo de la cultura. Esto ha sido decisorio para los sectores hegemónicos dominantes y en razón de ello es que existe la necesidad de controlar los medios de producción ideológicos. Ahora bien, es importante describir cuál es el papel del sector hegemónico que lucha frente a estos mecanismos en la disputa por el poder: este sector debe asumir una doble articulación si quiere posicionarse como dominante. Se trata de un principio de giro económico y de liderazgo cultural que ha sido resultado de procesos con objetivos mixtos (políticos, económicos y culturales) y que han permeado en las prácticas sociales. De esta manera, el texto ofrece la posibilidad de entender el ejercicio del poder en un espacio no esencialista, que le da un lugar de enunciación al sujeto en la praxis y permite apuntar y configurar la voluntad colectiva que represente los intereses económicos de la clase dominada.

Se reconoce entonces que aunque los grupos hegemónicos forman culturalmente la sociedad para perpetuar el poder, dentro de la ética militar se pueden generar puntos de irrupción en relación con la formación de intelectuales orgánicos que contribuyan al reconocimiento de sectores subordinados en función de la protección, las garantías y la restitución de los derechos a la sociedad civil. Esta formación intelectual que plantea Mouffe (1991) en el contexto de la quinta generación es posible dentro del marco de las Tic, las cuales han tomado fuerza en el último lustro en torno a los movimientos sociales, las reformas educativas y el reconocimiento de grupos invisibilizados. 
Reformar el primer momento educativo en torno a la reestructura de los intelectuales orgánicos ya tiene consigo una ventaja, y es que las Fuerzas Armadas no tienen que deliberar el liderazgo cultural, dado que ya forman parte de él.

Dicho esto, se extiende la invitación a que más allá de una clase de ética magistral, se demande la fundamentación sustentable para la protección del pueblo por medio de la ética y la moral. Es importante entender que el progreso militar no implica necesariamente una formación íntegra, de manera que es necesario suscitar el concepto de intelectuales orgánicos para reconocer en la población las necesidades y no invisibilizar los hechos de violencia, miseria e injusticia. De esta manera, es necesario intensificar un punto clave para la deconstrucción del progreso militar sin sensibilidad humana: el reconocimiento de la población y sus necesidades.

Para ello, el profesor Wilmer Villa (2013) traza una línea diacrónica y sincrónica de las categorías y procesos históricos que repercutieron en la identidad nacional desde el periodo de la Conquista. El artículo desarrolla las formas de invención que intervinieron en la construcción de la diferencia y cómo estas marcan las categorías sociales de lo aceptado y lo negado a lo largo de la formación discursiva. Es de vital importancia referenciar el artículo, dado que es posible sustentar cómo por medio de las intervenciones sociales se recrean conductas de invisibilización y negación y cómo por medio de la ética militar es posible lograr el reconocimiento con una postura crítica y decolonial.

El imaginario se configura por medio de la construcción de la identidad nacional, sustentada en una serie de relatos que sujetan la afirmación de un nosotros en oposición de unos otros, entendiendo el nosotros como una instancia de identidad que se considera superior a los otros y que pretende imponer sus prácticas sobre las de los otros, grupo que representa el sector hegemónico dominado (Mouffe, 1991), para integrarlos al considerarlos como inferiores y seres que requieren ser educados y amoldados a las prácticas que se agenciaron desde la posición de poder y privilegio de un nosotros. Es importante entender que el proyecto de conformación de una identidad nacional es un proceso a largo plazo y que es el resultado de perpetuar y reproducir ciertas prácticas desde el proceso de configuración del imaginario colectivo sobre la consideración del otro como inferior. 
La categoría del nosotros se genera a partir de la unión colectiva de un yo que se posiciona desde un marco de superioridad en oposición al otro que es diferente. Esta configuración del nosotros se da a partir de la movilización de imaginarios de mundo, es decir, desde el proceso colonial se crea esa noción de superioridad y de diferencia con respecto al otro, y es desde allí que se posiciona para intentar colonizar al otro y tratar de meterlo en sus dinámicas, con lo cual niegan la posibilidad de que el otro se posicione desde su propia voz.

Como segundos sujetos implicados se encuentra la configuración de los otros, que se instaura como categoría en oposición al nosotros que se encuentra en el centro, es decir, aquellos que ostentan el poder. La configuración de la categoría de los otros se da en el marco de los imaginarios colectivos que pretenden crear una identidad nacional, como un grupo que debe ser eliminado y cuyas prácticas deben ser negadas por no considerarse acorde con lo que se plantea desde el centro.

El posicionamiento dominante de un grupo que ostenta el poder se da mediado por formas de producción que permiten la reproducción, con el fin de establecer una reafirmación identitaria. En ese sentido, el actor del discurso disciplinar es el poseedor de lo que se considera el conocimiento o la verdad desde una posición de centro y, por ende, aquel que establece unas formas de producción de conocimiento que se consideran válidas. En este punto es importante entonces mirar el discurso disciplinar desde la escuela y, específicamente, desde el marco militar. Al cuestionar si esta institución replica nociones de verdad sustentada en un nosotros, es posible recurrir a una transformación en sentido del reconocimiento a la totalidad de la población civil, de tal manera que se garantice el derecho a la diferencia en todos los sentidos.

$\mathrm{Al}$ situarse en este contexto, la guerra de quinta generación también establece una serie de artefactos que se perpetúan en el espacio del conflicto, una invisibilización de grupos considerados como diferentes. Así, los artefactos y las mediaciones están enfocados a la forma de representación colectiva que sujeta el grupo dominante por medio de un proceso de fijación y distribución que permite representar la sociedad imaginada mediante procesos como la pedagogización —en la cual, como ya se dijo, se busca homogeneizar-y de 
rejillas de apropiación, que son producciones narrativas maestras que actúan en la clasificación y la generación de discursos (Villa, 2013).

En este contexto se entiende que las revistas, los periódicos y las recreaciones literarias generan discursos de dominación e invisibilización por medio de la reproducción cultural, que también se dan en la guerra de quinta generación respecto al tema de la información, dado que esta tiende a desestabilizar a las naciones. La opinión colectiva se posiciona en función de la cantidad de información generada en medios de comunicación o redes sociales. Dicha información puede ser veraz, verosímil, adulterada, tergiversada o deliberadamente falsa, lo que se conoce comúnmente como fake news.

Una vez expuesta la segunda propuesta teórica desde la que se deben pensar las reformas educativas, a continuación se expone el último punto, en el cual se relacionan las Tic en la democratización de la información y la creación de tejidos conectivos, reconocimiento de los pares y apropiación de las tecnologías en función de las prácticas éticas. Con este propósito se toma al autor Pierre Lévy (2004), quien propone desde el punto de vista humanista el reconocimiento de las tecnologías como fuentes de oportunidades en los espacios políticos, educativos y culturales.

El proceso de la globalización ha traído varias posibilidades interesantes, como el acceso a la información, la comunicación inmediata, la distribución de la producción académica, la generación de debates y de espacios de aprendizaje como las clases virtuales. El uso de las Tic en el entorno escolar, visto desde esta paleta de beneficios, permite el fácil acceso a la información y su manejo, además de que permite potenciar las mediaciones didácticas que vinculan la relación entre docente y estudiante.

En su apartado sobre el ciberespacio, Pierre Lévy (2004) se enfoca en un concepto que la época de la globalización ha escondido por su grado de peligro inminente: las humanidades. Según el autor, el neoliberalismo ha traído consigo una serie de prácticas deshumanizadoras que repercuten en la subjetividad e intersubjetividad humanas. De acuerdo con este autor, las tecnologías han sido un medio para promocionar y justificar corrientes frívolas que, por medio de dinámicas comunicativas (que están más allá de las relaciones inter- 
personales), transgreden a los negocios de mercadeo y publicidad, al tiempo que promueven la identidad social a un marco de consumo y competencia.

Por otro lado, Lévy (2004) entiende el carácter vitalicio de las humanidades en la virtualidad y la desarrolla dentro de un espacio íntegro y sin fronteras, donde la herramienta se vuelve foco transversal de oportunidades y donde los puntos de opresión se unen con el fin de compartir el conocimiento. Lévy (2004) considera que el auge de las innovaciones tecnológicas y la era digital tienen varios matices y tonos de conocimientos que están prestos a infinidad de variables, entre las cuales no se puede olvidar la dimensión humana, dado que esta construye civilización. Desde esta perspectiva, la tendencia a generar múltiples perspectivas descentraliza el conocimiento y logra evacuar la monopolización que han tenido históricamente las ciencias.

El enfoque inmanente del saber logra revocar las conductas excluyentes en cuanto recibe el pensamiento como un hecho autónomo. Ahora bien, la formación ciudadana en el ciberespacio al servicio del conocimiento colectivo le da un papel fundamental al educador de las humanidades, dada la labor que tiene en la promoción de canales colaborativos para la construcción de conocimiento. Las dinámicas con las cuales se inscribe la sociedad en el marco de la internet deben contemplar la formación ciudadana, de modo que el ciberespacio ha permitido la llamada cibercultura (Lévy, 2004): "Podría convertirse en un medio de exploración de los problemas, de discusión pluralista, [...] de hacer visibles procesos complejos, de toma de decisión colectiva y de evaluación de los resultados cercanos a las comunidades en cuestión" (Lévy, 2004, p. 41).

El entendimiento de la teoría humana en el ciberespacio es importante para el proyecto de la quinta generación, en el sentido en que da cuenta de la simultaneidad de usos pedagógicos que se pueden desarrollar por medio de la tecnología. Asimismo, es menester considerar que el medio en el cual se está desarrollando la cultura y la sociedad no es un enigma, sino un recurso para la transversalidad de conocimiento y el reconocimiento social e histórico, que se debe configurar a través de la adquisición como personas políticas para el tejido de una sociedad llena de intelectuales orgánicos y no clausurados, como los denominaría el autor. En ese sentido, la aplicación de la ética militar 
en el marco de las Tic toma una voz principal, en cuanto recurre al uso de ellas para conducir a una formación política del cuerpo armado para reconocer por medio de la responsabilidad los juicios, valores y proyectos éticos que la institución busca enaltecer, como la democracia directa, por medio del nuevo campo de batalla que recrea la quinta generación. En función de esto, el autor propone que

un dispositivo de democracia directa en tiempo real en el ciberespacio permitiría a cada cual contribuir continuamente a elaborar y a refinar los problemas comunes, a introducir nuevas preguntas, a forjar argumentos, a enunciar y adoptar posiciones independientes unas de otras sobre una gran variedad de temas. (Lévy, 2004, p. 44)

Así, más que herramientas de uso necesario, las Tic son un espacio potencial de encuentro en el que se suspenden los intereses individuales, las jerarquías y el intercambio de bienes (materiales) para privilegiar los intereses comunes, cuyo discernimiento está determinado por las formas de argumentación: "El uso socialmente más útil de la informática de comunicación es sin dudas proporcionar a los grupos humanos los medios para mancomunar sus fuerzas mentales para constituir colectivos inteligentes y hacer vivir una democracia en tiempo real" (Lévy, 2004, p. 42).

La inteligencia colectiva no es, entonces, una forma de agrupación común o estándar mediada por tecnologías, sino, según Lévy, la forma de agrupación cooperativa que "debe ser concebida como un verdadero servicio público", pues ella abre las posibilidades a una verdadera forma de democracia, la democracia directa.

La deconstrucción del imaginario colectivo en torno a las Fuerzas Militares tiene que verse como un diálogo en el que ambas partes (sociedad y cuerpo militar) ceden para buscar un liderazgo cultural. En ese sentido, la propuesta teórica sustentada por los tres autores resulta viable en el marco educativo, dado que amplía su mirada desde un punto de vista histórico, contextual y sociológico. Por su parte, la primera proposición busca fundamentar que la posibilidad de un cambio social, sustentado en el interés de los grupos que históricamente han sido subordinados, es posible por medio de la formación 
ética e ideológica de los líderes que reproducen la ideología, en este caso, del grupo dominante de las Fuerzas Armadas estatales.

El segundo corpus teórico dirige su perspectiva a recrear las formas de invención discursiva que distancia el sector hegemónico dominante del dominado. Aunque ya fue expuesto, resulta trascendental reconocer que existen producciones ideológicas en el marco de la guerra de quinta generación que transgreden la ética militar, por ello el segundo elemento es fundamental para permitir la extensión de un marco reflexivo y crítico en torno a las formas de dominación y representación por parte de los actores directos de la guerra.

El tercer punto centra sus esfuerzos en reconocer que aunque el modelo de la libre consecuencia ha traído consigo grandes atropellos a la sociedad civil, también es un puente de creación en torno a la defensa de la democracia directa y participativa. En ese sentido, la implementación de las Tic se debe generar desde el espacio político y ético, de manera que permita al cuerpo militar reconocer estas formas de participación en las que la soberanía y el bienestar social se inscriben en el marco del ciberespacio.

\section{Conclusiones}

$\mathrm{Al}$ entender las guerras de quinta generación es posible cuestionar, problematizar y proponer cuáles son los factores de carácter social, cultural y político que se afectan de forma directa a manos de los actores de dichas confrontaciones. Es importante entonces reconocer el papel protagónico de la ética militar como posible camino dentro de la trazabilidad de la protección civil, en aras de promover el respeto, la defensa de las prácticas soberanas, el reconocimiento a la diferencia y la participación social. Para ello resulta casi que imperante reestructurar las formas educativas en las que el cuerpo militar se inscribe en el campo de la guerra. En consecuencia, se reitera la formación ética como pilar fundamental en la trazabilidad de este proyecto.

También es importante problematizar el papel del cuerpo en el marco del conflicto de guerras de quinta generación en relación con la formación de la identidad, entendida como un proceso-producto de reproducciones subjetivas en el marco de la intersubjetividad que se legitima por medio del lenguaje. 
De acuerdo con Mudimbe (2010), la identidad es sobre todo una percepción posibilitada por los órganos del cuerpo, de manera que la experiencia del yo con el otro se construye a partir de lo corpóreo; el cuerpo, además, es el que interpreta las relaciones de identidad social que permiten afirmarse en el nosotros, establecido frente a grupos exteriores. Es decir, una relación con los otros y consigo mismo, mediado por la experiencia lingüística que permite al ser humano ser pasible de una identidad, entendiendo que desde que nacemos ya estamos inmersos en un mundo como significante y que dentro de la experiencia semiótica se crea la identidad.

Reconocer la identidad como una cadena de significantes en la que el yo y el otro se satisfacen mutuamente establece un sentido fundamental en relación con las guerras y las relaciones sociales, dado que ellas en sí son construidas mediante un acuerdo lingüístico que genera la identificación. Este acuerdo se da entre una identidad colectiva (estructurada o impuesta) y una identidad individual, de modo que los procesos de producción que se desarrollan en el marco de la cultura de la representación son empleados desde este marco y son atravesados por una interposición económica, política y ética.

La quinta generación de la guerra se debe pensar en el sentido de la configuración identitaria, dado que, como se dijo, en el ámbito de las relaciones sociales constituye el puente dialógico de las confrontaciones. Entonces, si se piensa al sujeto perteneciente al cuerpo militar desde una perspectiva ética, esta se expresa en una condición dual: por un lado, se establece en relación consigo mismo, es decir, el modo en que el sujeto configura y entiende la manera como se debe relacionar con los otros, que vendría a entenderse como los dilemas impuestos por las funciones dominantes. Por otro lado, la configuración de las conductas que la persona asume como correctas y que generalmente están ligadas con el comportamiento de los demás, lo cual está relacionado con la percepción del entorno que en efecto tiene un rol condicionante en el actuar del individuo.

Otra conclusión importante tiene que ver con que las formas de producción históricas, económicas y culturales han modificado las formas de vida y, consecuentemente, las dinámicas de la guerra. Al reconocer el contenido cambiante de las sociedades que están marcadas por el modelo neoliberal, es 
posible proyectar modelos educativos capaces de confrontar aparatos ideológicos basados en el control y el beneficio minoritario. En ese sentido, la ética profesional no solo extiende su importancia en el campo del cuerpo armado, sino que además incumbe a la mayoría de los procesos relacionados con las prácticas de saberes; así pues, la educación se entiende como la mayor fuente de configuración ética y ciudadana.

Al proponer que el marco de la ética militar es fundamental para un espacio de guerra, y luego de problematizar la reproducción de saberes basados en la negación del otro, es menester también concluir que la educación debe darse desde una mirada decolonial. En ese sentido, esta conclusión está enfocada en las dinámicas que extienden el marco problémico en relación con la formación ética de la educación. La relación hegemónica del saber-poder que ha sido constituida en las instituciones educativas trabaja en función de un desarrollo colonizador, en el cual la diferencia es una práctica de distanciamiento que establece relaciones de intersubjetividad y subjetividad. Esta es la forma en que se jerarquiza el conocimiento y se desarrolla en instancias máximas de representación en función de la utilidad y los marcos de condiciones sociales occidentales.

Por último, la investigación realizada evidenció que en el contexto de las guerras de quinta generación se recurre a dinámicas totalmente diferentes a las conocidas. Al respecto, es importante señalar que algunas prácticas de esta generación están mutando en Colombia, tales como las tácticas de rastreo de información personal de la ciudadanía. No obstante, esta capacidad tecnológica no trasciende en comparación con los otros países de primer mundo, lo cual puede ocurrir porque Colombia también responde a dinámicas de dominación externas en relación con el primer mundo, condición que ubica a Colombia en intereses más primarios con el fin de satisfacer la primera etapa de producción de los países en vía de desarrollo. Este último momento de reflexión pretende dejar abierta una pregunta en relación con ese desarrollo atrasado de las tecnologías bélicas: ¿Resulta positivo en un contexto como el colombiano el desarrollo de armas bélicas considerando el estado ético de las instituciones nacionales? 


\section{Referencias}

Althusser, L. (1988). Ideología y aparatos ideológicos del Estado. Freudy Lacan. Nueva Visión. Alderman, R. (2015). Defining fifth generation warfare. Military Embedded Systems.

Álvarez, C., Santafé, G., \& Urbano, O. (2017). Metamorphosis bellum: ¿¿Mutando a guerras de quinta generación? En C. Álvarez (ed.), Escenarios y desafíos de la seguridad multidimensional en Colombia (pp. 145-248). Escuela Superior de Guerra "General Rafael Reyes Prieto". https://doi.org/10.25062/9789585652835

Antoniou, C. T., \& Reisman, W. M. (1994). The laws of war: A comprehensive collection of primary documents on international laws governing armed conflict (1. ${ }^{\mathrm{a}}$ ed.). Vintage Books.

Añasco, M. T., \& Vera. P. (2019). Rol del trabajador social en procesos de empoderamiento de derechos de asistencia social a personas de escasos recursos de Machala. Unidad Académica de Ciencias Sociales.

Ávila, F. (2006). El concepto de poder en Michel Foucault. Telos, 2(8), 215-234.

Betancur, G. E. (2016). La ética y la moral: Paradojas del ser humano. CES Psicología, 9(1). 109-121. http://dx.doi.org/10.21615/cesp.9.1.7

Bermúdez, D. G. (2014). Comunicación y cibercultura: Video explicativo sobre la interrelación entre cultura, tecnología y música [Tesis de pregrado, Universidad Central del Ecuador].

Bruera, R. (2018). Los 14 puntos de Wilson: Una propuesta idealista en un mundo en guerra. Universidad Nacional de Córdoba.

Clausewitz, V. C., Howard, M. E., \& Paret, P. (1989). On war (Revised ed.). Princeton University Press.

De Vitoria, F., \& Pereña, L. (1989). Relectio de indis. CSIC-CSIC Press.

Díaz, S. R. (2015). Práctica de la ética en el desarrollo formativo de los estudiantes de una universidad privada de Chiclayo. Universidad Católica Santo Toribio de Mogrovejo.

Elliott, J. H. (1999). Europa después de la Paz de Westfalia. Pedralbes: Revista D’història Moderna, (19), 131-146.

Foucault, M. (2001). Un diálogo sobre el poder y otras conversaciones. Alianza.

González, C. N. (2017). Séptima generación de la guerra: Terrorismo como motor del reordenamiento de la seguridad. Boletín IEEE, (7), 515-526.

Gutiérrez, L. S. (2009). Alcances de una ética en el ciberespacio o el giro hacia una ética floreciente. Signo y Pensamiento, 28(55), 92-107.

Herrera, A. A. (2012). La noción de la guerra justa. Algunos planteamientos actuales. Analecta Política, 1(2). 273-290.

Herrera, A. A. (2009). La noción de seguridad en Thomas Hobbes. Revista Facultad de Derecho y Ciencias Políticas, (110), 97-124.

Lévy, P. (2004). Cibercultura: la cultura de la sociedad digital. Anthropos.

Lind, W. S. (2005). Compreendendo a guerra de quarta geração. Military Review, 85(3), 12-17. 
Lind, W. S. (2004). Understanding fourth generation war. Military Review, 84(5), 12-16.

Lind, W. S. (1985). Maneuver warfare handbook. Westview Press.

López, E., Hernández, E., \& García, O. P. (1999). Ética y economía: Desafíos del mundo contemporáneo. Plaza y Valdés.

Lozano, J., \& Parra, Y. (2015). La importancia de la ética y los DD. HH. en la formación de los alumnos de la Escuela Militar de Suboficiales "Sargento Inocencio Chincá. Universidad del Tolima.

Moliner, J. A. (2018). ¿Qué es la ética militar? Boletín IEEE, (11), 615-643.

Mouffe, Ch. (1991). Hegemonía e ideología en Gramsci. En H. Suárez, Gramsci y la realidad colombiana (pp. 167-227). Foro Nacional por Colombia.

Mudimbe, V. Y. (2010). ¿Qué es una línea? Sobre las paradojas en torno a las alegorías de identidad y alteridad. Boletín de Antropología, 20(37), 327-357.

Otero, H. (2012). La guerra en la sangre: Los franco-argentinos ante la primer guerra mundial. Sudamericana.

Poole, K. (2009). Foreword. En George A. Crawford, Manhunting: Counter-network organitatio for irrgular warfare. Join Special Operation University.

San Agustín. (1994). Obras completas de San Agustín (I: Escritos filosóficos: Introducción y biografía general. Vida de San Agustín escrita por San Posidio. Soliloquios. La vida feliz. El orden). Biblioteca Autores Cristianos.

Santo Tomás de Aquino. (1950). Metaphysics of Aquinas: A Summary of Aquinas's Exposition of Aristotle's Metaphysics. University Press of America.

Spinoza, B. (2019). Ética. LeBooks.

The White House. (2003, febrero 14). President Speaks at FBI on New Terrorist Threat Integration Center. https://georgewbush-whitehouse.archives.gov/news/releases/2003/02/20030214-5. html

Ureña, A. (2012). Blitzkrieg vs. batalla profunda. German Army Publishers.

Villa, W. (2013). Memoria y pedagogización del mal-decir: Una aproximación a los recorridos literarios que inventan mundos. Cuadernos de Filosofía Latinoamericana, 34(108), 1-29. https://doi.org/10.15332/s0120-8462.2013.0108.03

Wilson, W. (1918). Nuestro programa es la paz mundial. Discurso de Woodrow Wilson ante el Congreso de EE. UU. 